\title{
Understanding undergraduate nursing students' knowledge of, and attitudes to, prenatal genetic screening in Malaysia: A preliminary study
}

\author{
Soon Lean Keng*, Pricilia Dew Maria Stephen, Lai Sue Yi \\ School of Health Sciences, Health Campus,Universiti Sains Malaysia,Malaysia \\ *Corresponding author E-mail: soonlk@kb.usm.my
}

\begin{abstract}
Background: Nursing students must be able to ensure that patients understand important health screening information. However, studies have reported a deficit in nursing students' knowledge of genetics and genetic disorders.

Aim: The purpose of this study was to investigate nursing students' knowledge of, and attitudes to prenatal genetic screening.

Methods: A descriptive, cross-sectional survey was conducted with final year undergraduate nursing students recruited from a tertiary teaching hospital in Kelantan, Malaysia. Thirty-four of the 37 students returned a self-administered questionnaire (response rate $=97 \%$ ) that assessed knowledge of, and attitudes to, prenatal genetic screening.

Findings: The majority of the respondents demonstrated an adequate level of knowledge regarding prenatal genetic screening $(65 \%)$, and $62 \%$ were more supportive than others of screening. Approximately one third $(35 \%)$ had limited knowledge. Simple linear regression analysis uncovered a significant linear relationship between the attitude score and the knowledge score $(\mathrm{R} 2=0.48, \mathrm{P}<0.05,95 \%$ confidence interval).

Conclusions: These findings suggest the need to improve and upgrade the nursing education strategy concerning genetics. The deficit in nursing students' knowledge of prenatal genetic screening may result in inappropriate counselling, health teaching and referrals, which can have serious repercussions.
\end{abstract}

Keywords: Prenatal genetic screening, undergraduate nursing students, knowledge, attitude, Malaysia.

\section{Introduction}

Today's healthcare environment is rapidly becoming increasingly complex. The complexity of healthcare terminology, devices, and instructions on screening options can be overwhelming for patients who must navigate this complex system, make informed healthcare decisions, and care for acute or chronic health conditions at home. The practice of medicine is changing as clinical applications based on genetic technologies continue to emerge, with profound implications for nursing education and the nursing profession [2]. Despite the scientific world now understanding a great deal about genetics, public understanding of genetic information and screening has not kept pace [16].

An early study by Brantl and Esslinger [3] addressed the need for integrating human genetics into the nursing curriculum to prepare graduates with essential competencies. More recent surveys have indicated the need for integration of genetic and genomic information into the curriculum [4, 8, and 12]. Subsequently, researchers have stated that knowledge of genetics and genetic education is a critical aspect of academic nursing education [9, 11]. There have been calls for the acquisition of a greater fundamental understanding of genetic and genomic information among health professionals and graduate nursing students [10,11].

Despite recognition of the need for education in genetics [15], surveys have indicated that genetic content is still lacking in nursing schools' curricula [6]. This is of concern because nurses, in the frontline of patient care, are in a unique position to provide a supportive role in educating patients about, and motivating them to undergo prenatal genetic screening in primary health care settings. Therefore, it is important to understand undergraduate nursing students' knowledge of, and attitudes to, prenatal genetic screening because they are the nurses of the future. They must have the ability to recognize disorders, address patients' questions about genetic diseases, and inform patients about genetics and prenatal genetic screening. In Malaysia, however, there does not appear to have been any research in this area. This pilot study aimed to raise awareness of these issues by investigating undergraduate nursing students' knowledge of, and attitudes to, prenatal genetic screening. 


\section{Methods}

A descriptive cross-sectional study was conducted in the School of Health Science of a tertiary teaching hospital in Kelantan. The study had the approval of the Dean, School of Health Science and the Human Research Ethics Committee. The participants were final year degree-level nursing students studying in a four-year bachelor's degree program. Informed written consent was obtained from each participant. They were informed that the whole exercise was confidential, anonymous, voluntary and purely for academic purpose. All 37 final year undergraduate nursing students of the School were included in the study. The researcher distributed the three-part self-administered questionnaire to all the students simultaneously after their regular morning clinical session in the health campus. For the students who were in the afternoon session of the survey, the questionnaires were circulated on subsequent morning days. A total of 34 participated and completed the questionnaire (response rate $=97 \%$ ).

\subsection{Instrument}

The questionnaire was developed from the available literature and included a series of items divided into three sections: (1) demographic characteristics (age, ethnicity and religion); (2) knowledge about prenatal genetic screening; and (3) attitudes toward prenatal genetic screening. The series of answers to the knowledge questions consisted of dichotomous items to which participants indicated their agreement with "true" and "false" choices. The score for knowledge regarding prenatal genetic screening was obtained by adding correct responses to all 22 items in this section, with scores ranging between 0 and 22. Higher scores reflected a higher knowledge level about prenatal genetic screening $(\geq 18)$ [5]. Ten items examined the student nurses' attitudes to prenatal genetic screening. Level of agreement or disagreement was rated on a five-point Likert-type scale ranging from "1" to "5", in which "1=unsure", "2=strongly disagree", " $3=$ disagree", "4=agree" and " $5=$ strongly agree". The total score for 10 items ranged from 1 to 50 , with higher scores indicating a more supportive attitude towards [14]. Experts in health sciences validated the questionnaire for face, content and construct validity. It was considered reliable, having a reliability of $\alpha=0.64$ [1].

\subsection{Statistical analysis}

Data collected were entered and analysed in the Statistical Package for Social Sciences (SPSS) version 19 software. The categorical variables were expressed in frequency and percentage. Simple linear regression was used to measure the relationship between the independent variable (the knowledge score) and the dependent variable (the attitudes score). Pvalues of 0.05 or less were considered statistically significant.

\section{Results}

\subsection{Demographic characteristics}

All participants were female. The majority (73.5\%) were Malay, 23.5\% were Chinese and 2.9\% were Indian. The maximum age of participants was 34 years and the minimum age was 22 years. Hence, the mean age was 23.29 years. The majority $(73.5 \%)$ followed Islam, $8.8 \%$ were Buddhist, $14.7 \%$ were Christian and $2.9 \%$ followed other faiths (Table 1).

\begin{tabular}{cccc}
\multicolumn{4}{c}{ Table 1: Participants' demographic characteristics (n=34) } \\
\hline Characteristics & Frequency & Percentage (\%) & Mean (SD) \\
\hline Age (Years) & & & $23.29(2.34)$ \\
Age (Years) & & 32.4 & \\
22 & 11 & 52.9 & \\
23 & 18 & 8.8 & \\
24 & 3 & 5.8 & \\
$>25$ & 2 & 73.5 & \\
Ethnicity & & 23.5 & \\
Malay & 25 & 2.9 & \\
Chinese & 8 & & \\
Indian & 1 & 73.5 & \\
Religion & & 8.8 & \\
Islam & 25 & 14.7 \\
Buddhist & 3 & 2.9 \\
Christian & 5 & & \\
Other & 1 & &
\end{tabular}




\subsection{Knowledge scores}

Results for the knowledge questions indicate that 22 participants scored 18 and above, while the remaining 12 participants scored 17 and below (see Table 2). Thus, 65\% of the participants had adequate knowledge of prenatal genetic screening (PGS). However, only approximately $5.9 \%(n=2)$ correctly answered all 22 questions.

\begin{tabular}{ccc}
\multicolumn{3}{c}{ Table 2: Knowledge score for prenatal genetic screening $(\mathrm{n}=34)$} \\
\hline Knowledge score & Frequency & Percentage $(\%)$ \\
\hline 15.0 & 3 & 8.8 \\
16.0 & 4 & 11.8 \\
17.0 & 5 & 14.7 \\
18.0 & 4 & 11.8 \\
19.0 & 8 & 23.5 \\
20.0 & 3 & 8.8 \\
21.0 & 5 & 14.7 \\
22.0 & 2 & 5.9 \\
\hline
\end{tabular}

Knowledge scores ranged from 15-22. Distribution was normal, with a mean score of 18.44 (see histogram, Figure 1).

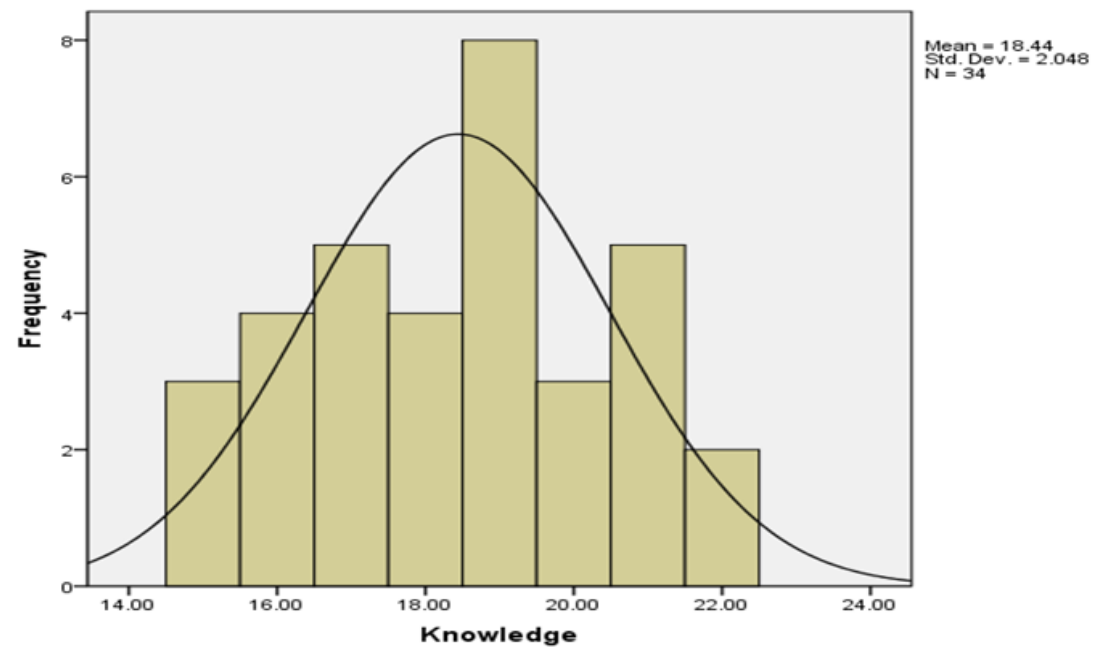

Fig. 1: Knowledge score for prenatal genetic screening $(n=34)$

\subsection{Attitudes scores}

On the attitudes score, 21 participants scored 35 and above. Support for prenatal genetic screening was evident in $62 \%$ of the participants. Thirteen participants scored less than 35, indicating that they were less supportive of prenatal genetic screening (see Table 3).
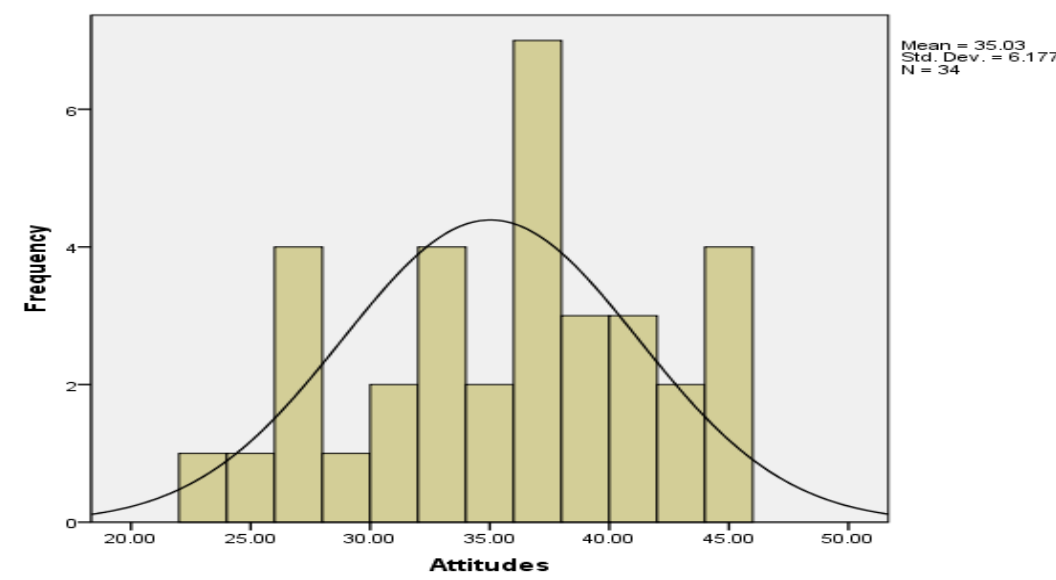

Fig. 2: Attitudes score for prenatal genetic screening $(n=34)$ 
Table 3: Attitudes scores for prenatal genetic screening $(\mathrm{n}=34)$

\begin{tabular}{ccc}
\hline Attitudes score & Frequency & Percentage $(\%)$ \\
\hline 23.0 & 1 & 2.9 \\
24.0 & 1 & 2.9 \\
26.0 & 2 & 5.9 \\
27.0 & 2 & 5.9 \\
29.0 & 1 & 2.9 \\
30.0 & 2 & 5.9 \\
32.0 & 3 & 8.8 \\
33.0 & 1 & 2.9 \\
35.0 & 2 & 5.9 \\
36.0 & 6 & 17.6 \\
37.0 & 1 & 2.9 \\
38.0 & 3 & 8.8 \\
40.0 & 3 & 8.8 \\
42.0 & 1 & 2.9 \\
43.0 & 1 & 2.9 \\
44.0 & 2 & 5.9 \\
45.0 & 2 & 5.9 \\
\hline
\end{tabular}

The distribution of scores was examined for normality and skewness. Distribution was normal, with a mean score of 35.03 indicating a range of scores from 23-45 (see histogram, Figure 2).

\subsection{Associations between knowledge and attitudes towards prenatal genetic screening}

The association between the students' knowledge and attitudes towards prenatal genetic screening was tested using simple linear regression. The scatterplot graph for the relationship between the two quantitative variables shows the independent variable knowledge score on the horizontal x-axis, and the dependent variable attitudes score on the vertical y-axis (Figure 3).

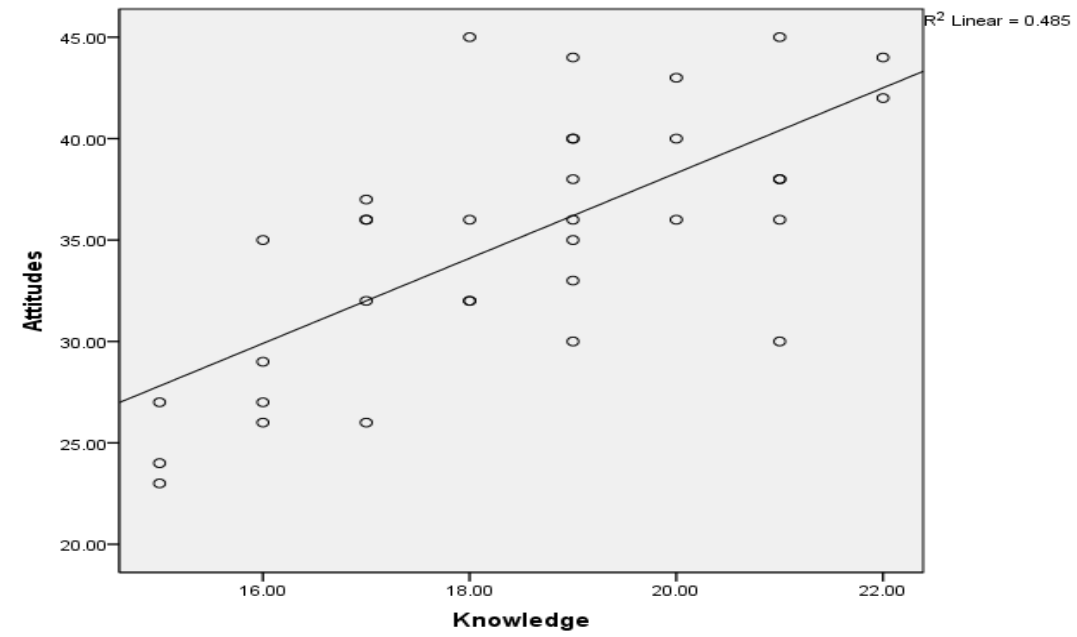

Fig. 3: Attitude score of prenatal genetic screening against knowledge score of prenatal genetic screening $(n=34)$

Simple linear regression measured the association between the knowledge score and the attitudes score (see Table 4).

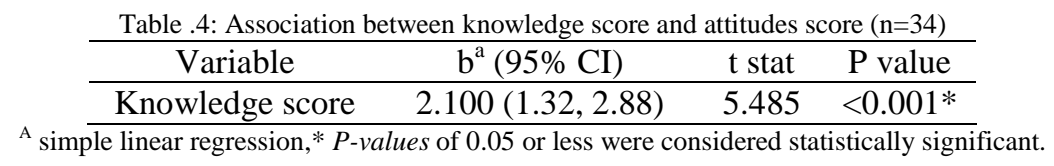

Figure 3 and Table 4 shows that the knowledge score explains $48 \%$ of the variation in the attitudes score. The attitudes score is significantly and linearly related to the knowledge score $(\mathrm{p}=<0.001)$. Individuals with 1 score higher knowledge has 2.1 score supportive attitudes towards prenatal genetic screening. In the population, with 1 score change in 
knowledge score, the change in the attitudes towards PGS ranges from 1.32 to 2.88 points with $95 \%$ confidence (Table 4).

\section{Discussion}

The results show significant gaps in nursing students' reported knowledge of prenatal genetic screening and indicate the need for nursing students to develop their knowledge of prenatal genetic screening further. As noted, almost one third of the nursing students had inadequate knowledge about prenatal genetic screening. A likely explanation for poor knowledge amongst these students is the exclusion of reference to prenatal genetic terms and concepts in their university courses. The lack of high levels of knowledge can be seen as an indication of students' inability to provide sound medical knowledge. This is a matter of great concern.

The correlation between knowledge about genetics and attitude to prenatal genetic screening among survey participants indicates that improved knowledge should lead to improved attitudes to genetic screening. The results are similar to those of Kiray Vural et al.'s survey, which was conducted with 162 nursing students in Turkey. Kiray Vural et al. postulate positive correlation between the nursing students' level of knowledge and their degree of attitude towards genetics [9].

The current results also echo the findings of Jenkins et al. regarding the need for educational preparation in genetics for nurses [8]. This suggests that there may well be a ceiling effect for learning related to the present curriculum. This study lends support to the need for improved education and a changed curriculum. The clinical application of prenatal genetic knowledge has major implications for the nursing profession regardless of academic preparation, role, or practice setting. The public will increasingly expect that the nurse will use the prenatal genetic information when providing care. These expectations have direct implications for registered nurse preparatory curricula, as well as the practicing nurses. The survey shows that nursing students need to develop their knowledge of prenatal genetic screening in order to provide effective health teaching about it. This finding has implications for nursing education, pre-empting a call for curricular review so that prenatal genetic principles become part of the curriculum in university courses $[3,6,7$, and 15]. The increased demand for high-quality health care in the genomic era requires nursing students, who are the future front line health care professionals, to understand the relevance of prenatal genetics and the concepts of screening in practice. Similarly, Sobhy et al. found that nursing students who are well informed about genetic counselling services are better informed about genetic education and can provide genomics education to their patients [13]. The research findings emphasize the importance and urgency of education that focuses on both prenatal genetics and screening [12].

\section{Limitations}

This study was conducted at one tertiary teaching hospital in Malaysia. Thus, results may not be generalizable to other nursing programs. Despite this limitation, the study provides valuable subjective information concerning nursing students' knowledge of, and attitudes towards, prenatal genetic screening. It also provides new information concerning prenatal genetic education in the university curriculum.

\section{Conclusion}

It can be concluded that nursing students must understand the relevance of prenatal genetic screening to meet contemporary society's increased demands for knowledge of, and effective practice in, genomics. As a result, university nursing programs must reform current educational strategies concerning genetics and prenatal genetic screening. Genomics must be recognized as an important course of professional training at the undergraduate level.

\section{Acknowledgements}

This study would not have been possible without the funding support from the Universiti Sains Malaysia Postgraduate Incentive Grant Reference Number: 1001/PPSK/8123020 and all undergraduate nursing students directly or indirectly involved in this study. We also would like to thank the Dean, School of Health Science, Universiti Sains Malaysia for giving us permission to conduct this study. Finally, we would like to thank the contribution of Margaret Bowden, of Flinders University of South Australia, Australia in editing this manuscript. 


\section{References}

[1] Al-Saleh, K.S.M.Z. Ramadan. Direct and Indirect Employee Questionnaires for Assessing Patient Safety in Saudi Hospitals. Applied Medical Informatics. Vol. 26, No.2, (2011), 63-72.

[2] American-Nurses-Association, Essentials of genetic and genomic nursing: Competencies, curricula guidelines, and outcome indicators. 2008: Silver Spring (MD): American Nurses Association.

[3] Brantl, V.P. Esslinger. Genetics Implications for Nursing Education. Nursing Forum. Vol. 1, (1962), 90-100

[4] Calzone, K.A., A. Cashion, S. Feetham, J. Jenkins, C.A. Prows, J.K. WilliamsS.-F. Wung. Nurses Transforming Health Care using Genetics and Genomics. Nursing outlook. Vol. 58, No.1, (2010), 26.

[5] Gharaibeh, H., A. OweisK. Hamad. Nurses' and Midwives' Knowledge and Perceptions of Their Role in Genetic Teaching. International Nursing Review. Vol. 57, No.4, (2010), 435-442.

[6] Hetteberg, C.C.A. Prows. A Checklist to Assist in the Integration of Genetics into Nursing Curricula. Nursing outlook. Vol. 52, No.2, (2004), $85-88$.

[7] Hetteberg, C.G., C.A. Prows, C. Deets, R.B. MonsenC.A. Kenner. National Survey of Genetics Content in Basic Nursing Preparatory Programs in the United States. Nursing outlook. Vol. 47, No.4, (1999), 168-174.

[8] Jenkins, J.K.A. Calzone. Establishing the Essential Nursing Competencies for Genetics and Genomics. Journal of Nursing Scholarship. Vol. 39, No.1, (2007), 10-16.

[9] Kiray Vural, B.T., AG, Kurban NK \& Taspirnar, A. Nursing Students' Self-reported Knowledge of Genetics and Genetic Education. Public health genomics. Vol. 12, No.4, (2009), 225-232.

[10] Kirk, M., D. LeaH. Skirton. Genomic Health Care: Is the Future Now? Nursing \& health sciences. Vol. 10, No.2, (2008), 85-92.

[11] Prows, C.A., M. Glass, M. Nicol, H. SkirtonJ. Williams. Genomics in Nursing Education. Journal of Nursing Scholarship. Vol. 37, No.3, (2005), 196-202.

[12] Seibert, D., Q.T. EdwardsA. Maradiegue. Integrating Genetics into Advanced Practice Nursing Curriculum: Strategies for Success. Public health genomics. Vol. 10, No.1, (2006), 45-51.

[13] Sobhy, S., F. ShoeibN. Zaki. Assessment and Upgrading of Alexandria University Nursing Students' Knowledge and Attitudes about Genetic Counseling. The Journal of the Egyptian Public Health Association. Vol. 76, No.3-4, (2000), 205-222.

[14] Toiviainen, H., P. Jallinoja, A.R. AroE. Hemminki. Medical and Lay Attitudes towards Genetic Screening and Testing in Finland. European journal of human genetics. Vol. 11, No.8, (2003), 565-572.

[15] Tomatir, A.G., H.Ç. Sorkun, H. DemirhanB. Akdag. Nurses' Professed Knowledge of Genetics and Genetic Counseling. The Tohoku journal of experimental medicine. Vol. 210, No.4, (2006), 321-332.

[16] Williams, J.K., T. Tripp-Reimer, D. SchutteJ.J. Barnette. Advancing Genetic Nursing Knowledge. Nursing outlook. Vol. 52, No.2, (2004), 7379. 